\title{
Research and Design of Intelligent Products for Sitting Posture Correction
}

\author{
Jiaqi CHANG ${ }^{\text {a }}$, Yizhe LIU ${ }^{\mathrm{a}}$, Jiaqi LI ${ }^{\mathrm{a}}$, Yan $\mathrm{JIANG}^{\mathrm{b}}$ and Yi LIU ${ }^{\mathrm{a}, 1}$ \\ ${ }^{a}$ Fashion Accessory Art and Engineering College, Beijing Institute of Fashion \\ Technology, Beijing, P. R. China \\ ${ }^{\mathrm{b}}$ College of Arts and Sciences, Beijing Institute of Fashion Technology, Beijing, $P$. \\ R. China
}

\begin{abstract}
This paper analyzes the status and causes of improper sitting posture group, and compares the current situation of sitting posture correction products on the market. Based on the Lego EV3 model, the product design is implemented. Three intelligent correction schemes are proposed and analyzed. Finally, the prototype of the product is produced to achieve the purpose of intelligent detection and prompt users to correct the sitting position.
\end{abstract}

Keywords. Sitting posture correction; Intelligent products; Product design; Classroom Observation

\section{Status of sitting problems}

In information age, people spend more and more time sitting in front of computers for working, internet accessing, leisure and entertainment. When sitting time becomes longer and the posture will change too. There are some bad sitting postures that harm people's body that is not detected easily, which can lead to a variety of problems and physical diseases, such as hunchback, high and low shoulder and other physical problems, as well as myopia and other related diseases.

\subsection{Physiological characteristics of people with improper sitting posture}

\subsubsection{Body image issues}

The top five improper body postures in China are round shoulders, high and low shoulders, hunched back, razor back and probing neck. These postural problems often occur because of fixed postures, or prolonged poor postures that cause excessive tension in some muscles. In a short term, improper posture only affects aesthetics. But in the long term, poor posture can put the spine in a bad position and lead to muscle imbalance around the spine, which can cause muscle pain in mild cases and scoliosis, pelvic tilt and other spinal problems in severe cases. And indirectly affect cardiopulmonary function, digestive function, growth and development, and also have a negative psychological impact on people. 


\subsubsection{Related diseases}

Improper sitting posture can also indirectly cause myopia. A research report of the World Health Organization shows, in 2020, the number of global myopia patient is about 2.5 billion. In our country, it is amounts to 600 million. Adolescent myopia rate ranks the first place in the world, and presents the characteristics of small age, high incidence of disease, myopia degree deep.

During the prevention and control of COVID-19 period, large-scale online courses have brought new challenges to the prevention and control of myopia among children and adolescents. Myopia is mainly caused by the long-term improper sitting posture with poor eye hygiene, and with the growth of age and habit, if this problem cannot be corrected in time, it will become more and more serious.

\section{Sitting correction products status}

The traditional sitting posture orthotics on the market are divided into two categories: humpback orthotic braces (Figure 1) and anti-myopia table bracket (Figure 2). These two major categories is commercial available for a long time. product types are diverse, but this product correction is passive, lacking of scientific methods. Its advertising claims are worth more than the product itself, leading to the misconception that the use of the product can completely prevent hunchback. In addition, the use of these products requires scientific guidance, which is not readily available.

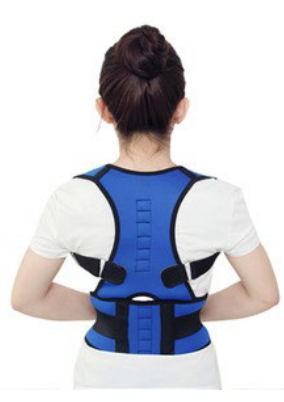

Figure 1. Hunchback correction back brace

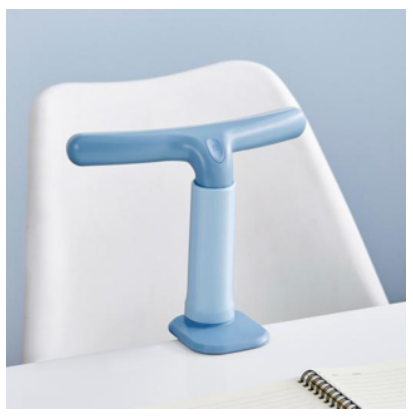

Figure 2. Anti-myopia desktop holder

Hump braces are a set of elastic straps worn on the upper back. It is not only inconvenient to wear, but also easy to cause prickly heat in summer. The products lack effectiveness. It can only slow down the early development of hunchback, but not play a correction and prevention effect. In addition, it should not be worn for a long time, because the correction of the neck and shoulder will compress the blood vessels in the body to some extent, then blocking blood flow.

The anti-myopia desktop bracket has great limitations on activities, such as affecting the action of turning pages; The effect is not good, the user can still bend over, head over the bracket; The fixture at the bottom of the bracket has requirements 
on the structure of the table, if there is no table edge will be difficult to fix; Stent height regulation lacks scientific standards.

Therefore, the correction product market now begins to develop toward the direction of intelligence, science and humanity, and begins to pay attention to the convenience of use and the scientific of the product itself. For example, distancesensing glasses are designed to correct wrong posture through sensors and judgment procedures, then prompt users to correct their sitting position.

\section{Research on product design for sitting posture correction}

\subsection{Physiological diagram analysis of correct sitting posture}

Proper sitting posture (Figure. 3) should include chin and head resting on shoulders in a vertical line with the spine. Shoulders relaxed, slightly spread back. Shoulder blades are contracted backward while arms are slightly turned outwards. The weight of the upper body falls on the ischium, and maintaining a certain degree of tightness in the waist and abdomen. The thigh is flat on the chair, and vertical with the upper body. The knee level is forward, and the angle between calf and thigh is 90 degrees, feet fall right in front of the knee, flat on the ground.

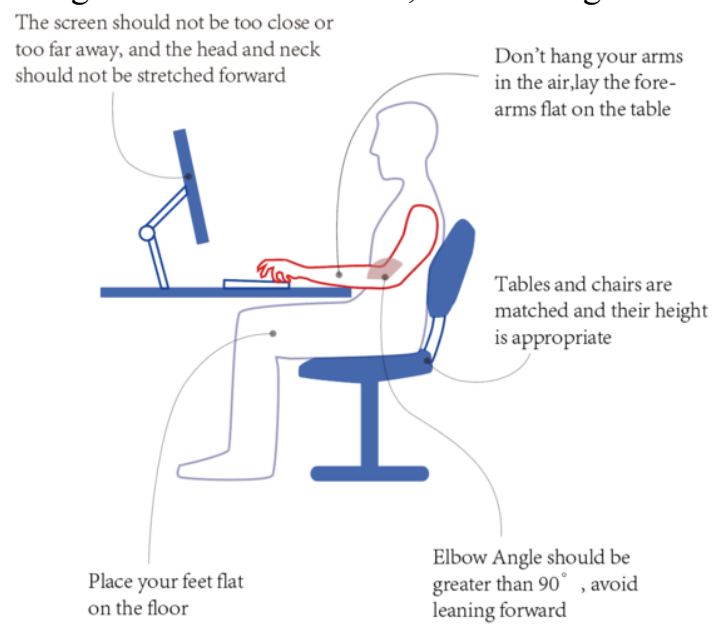

Figure 3. Diagram of proper sitting position

When user is reading, writing or using electronics on a desk, attention should be paid to the height of the desk so that the forearm can be placed flat on the desk and the angle between the upper and forearms should be greater than $90^{\circ}$. 


\subsection{Product proposal and analysis}

\subsubsection{Pressure sensing cushion (Figure 4)}

When sitting in an incorrect position, the pressure values in the legs and hips would change. For example, when the body is leaning forward or hunched over, the pressure peak will shift from the buttocks to the middle of the thigh.

When crossing the legs, the pressure on one side decreases, while the another side increases. Using the above principle, two sets of pressure sensors can be placed on the left and right sides of the cushion to monitor the change of pressure value in real time, so as to determine whether the sitting posture is correct. When the sitting posture is not correct, a vibration and sound alert is issued. It also has a timer function and gives a prompt when it detects that the user has been sitting for a long time or has overused the eyes.

The cushion not only detects and indicates the user's sitting posture, but also provides feedback on problems such as sedentary sitting, in addition to protecting the user's eye hygiene to a certain extent.

However, it is susceptible to environmental factors, the error is more difficult to offset, and for the sensor precision accuracy requirements are high, making the product cost significantly increased, consumer acceptance decreased.

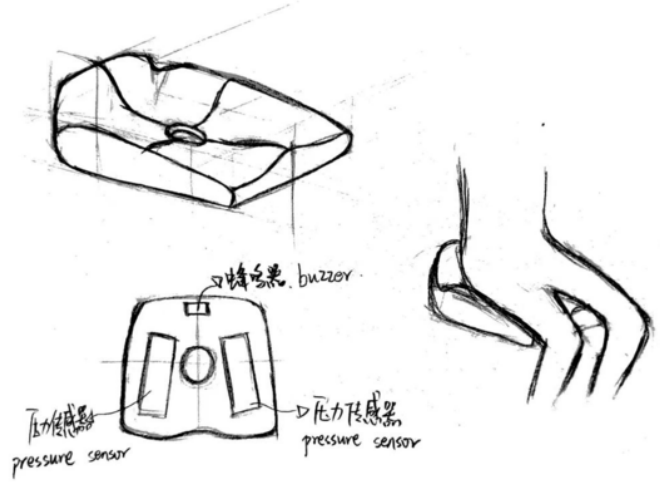

Figure 4. Schematic diagram of the pressure sensing cushion

\subsubsection{Spine tracking (Figure 5)}

The solution uses color sensors and ultrasonic sensors which are integrated into a seat cushion. The color sensors are arranged vertically in the center line of the cushion. When the spine is scoliosis, the upper and lower color sensors detect the difference in color and emit a vibration and an audible alert. The top of the cushion is an ultrasonic sensor to detect whether the sitting posture is tilted forward. If it is tilted forward, no distance is detected and a signal is sent to prompt the user to correct the sitting posture.

While this seat cushion is good at detecting spinal problems, it is ineffective at detecting poor sitting postures such as crossing the leg, which is somewhat lacking. 


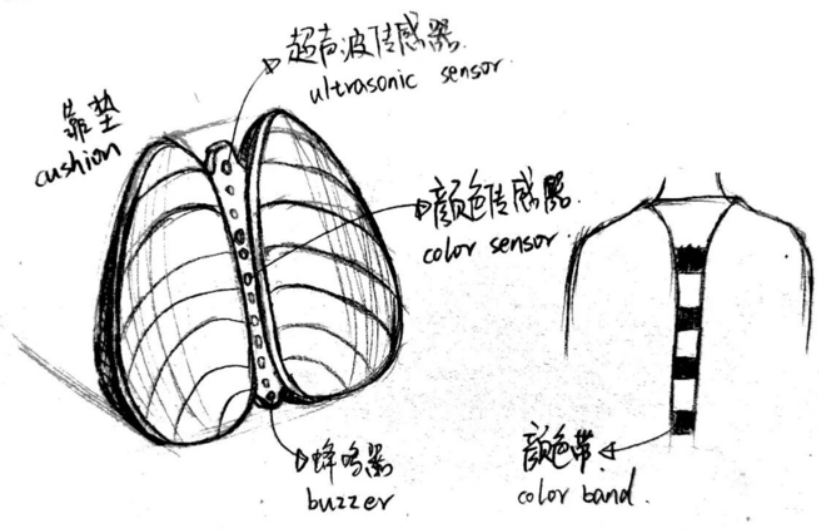

Figure 5. Schematic diagram of the spinal tracking

\subsubsection{Angular sensing and ultrasonic ranging terminal (Figure 6)}

Direct angle measurement is a simple but effective method. The angle sensor senses the upper arm and forearm angle, the upper body and thigh angle and the knee angle to ensure that it can be greater than or equal to $90^{\circ}$ to avoid forward leaning upper body and improper leg postures such as diagonal legs, crossed legs, etc. There is also a desktop ultrasonic rangefinder terminal used in conjunction with it, which is responsible for detecting the distance between the head and the rangefinder to determine whether the head is bowed, or pop the head out, etc. At the same time, as a terminal, it can sound an audible alert when sitting in an improper posture. It also has a timer function and gives a prompt when it detects sedentary behavior or excessive eye use.

With the detachable design, the weight of the wearable device is significantly reduced, making it more comfortable to wear. In addition, with the desktop terminal, multi-angle measurement is more accurate.

Its simple principle makes the product simple in construction and low hardware requirements while ensuring functionality, so it is easy to produce and sell, and easily accepted by consumers.

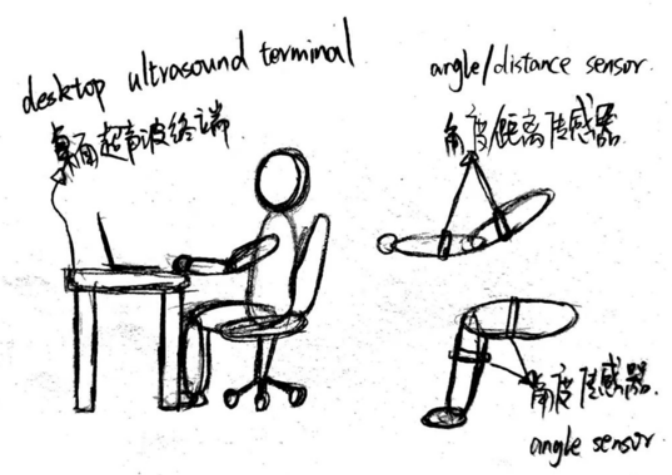

Figure 6. Schematic diagram of the angular sensing and ultrasonic ranging terminal 


\subsection{Implementation of in-depth programs (processes, procedures)}

The program consists of three basic structures: sequential structure, selective branch structure and loop structure (Figure 7). The sensor first receives two values, namely the angle on both sides, and determines whether it belongs to the threshold range for the first reminder. This judgment tests whether the sitting position is leaning forward or backward. Then wait for the user to correct the sitting position and judge whether the difference between the two values is greater than the threshold for a second reminder, this time to judge whether there is left-right tilt. The two judgments constitute a cycle structure, with a cycle interval of 10 seconds to reduce false positives caused by actions such as drinking water and taking things. The program takes 60 minutes as a cycle, prompting users to pay attention to rest.

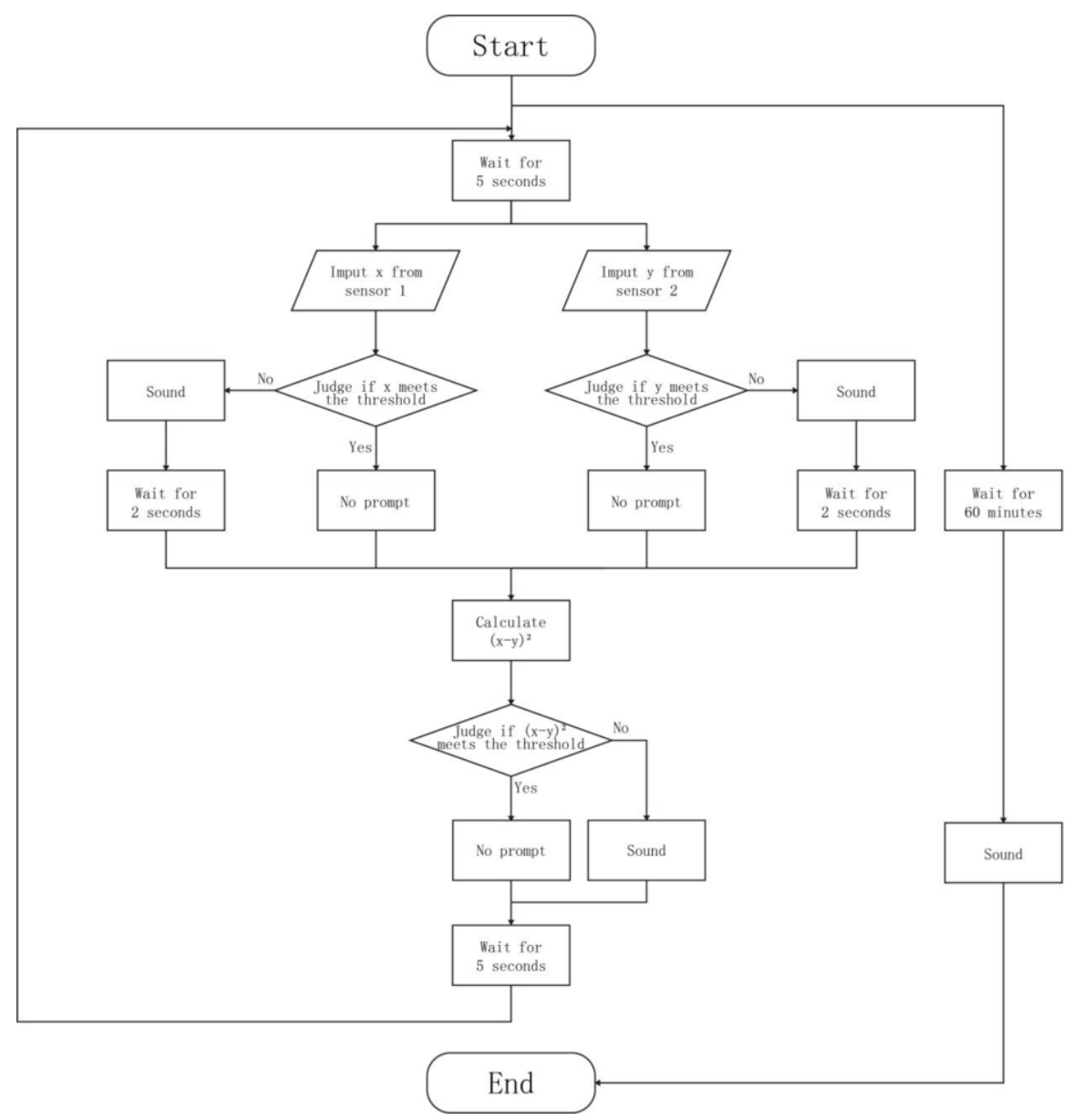

Figure 7. Program logic diagram 


\section{Prototype of EV3-based sitting posture correction product}

The prototype of this directional design product is shown in Figure 8, and the modules used are the motor and ultrasonic sensor. The program diagram is detailed in Figure 9.

\subsection{Active sitting alert device - elbow section}

The procedure is shown in Figure 9, where the standard angle is set by the body condition of different people according to the standard sitting posture that matches their body condition. The state of the arms is determined by detecting the motor angle, which further comes to determine the present sitting position of the body. If a single angle produces a range of change greater than (15), an alert is given, while if both elbows have the same range of change and are within $\left(30^{\circ}\right)$, no alert is given. The product also sets the detection time to every 10 s to ensure that the user has enough time to adjust the sitting position when they need to drink or get something. The device will also alert the user with an audible alert if the wrong sitting position is detected and the user does not adjust it in time.

\subsection{Active sitting alert device - crotch section}

This product is based on the same principle as the elbow angle detector. There is also an angle detection device at the crotch, which simply indicates if the angle change is in the range $\left(80^{\circ}-105^{\circ}\right)$ and also gives an alert if it is not.

\subsection{Active sitting alert device - knee section}

This product is based on the same principle as the elbow angle detection device. There is also an angle detection device at the knee, which simply indicates whether the angle change is in the range $\left(70^{\circ}-110^{\circ}\right)$, and also gives an alert if it is not in this range.
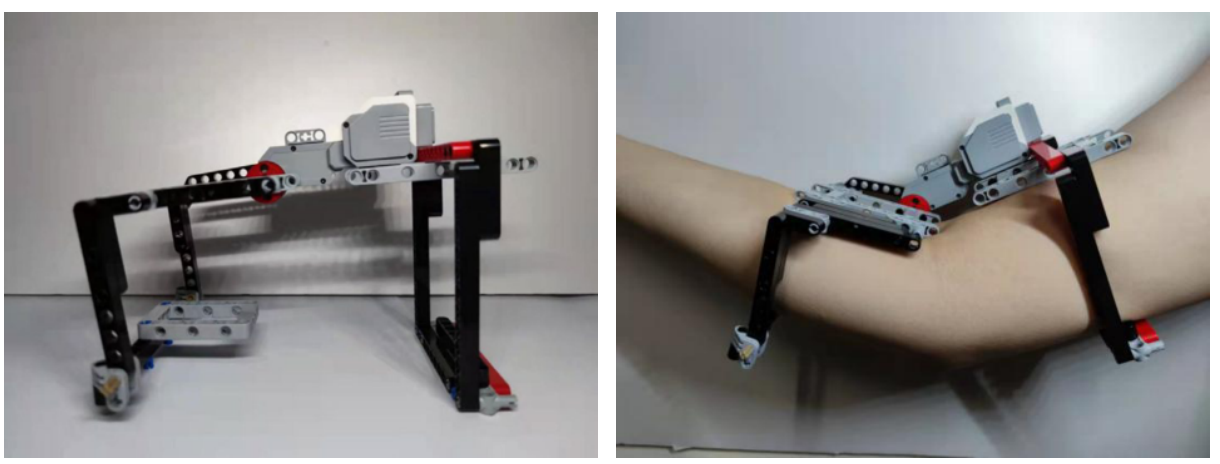

Figure 8. Prototype and use diagram of the sitting posture corrector product 


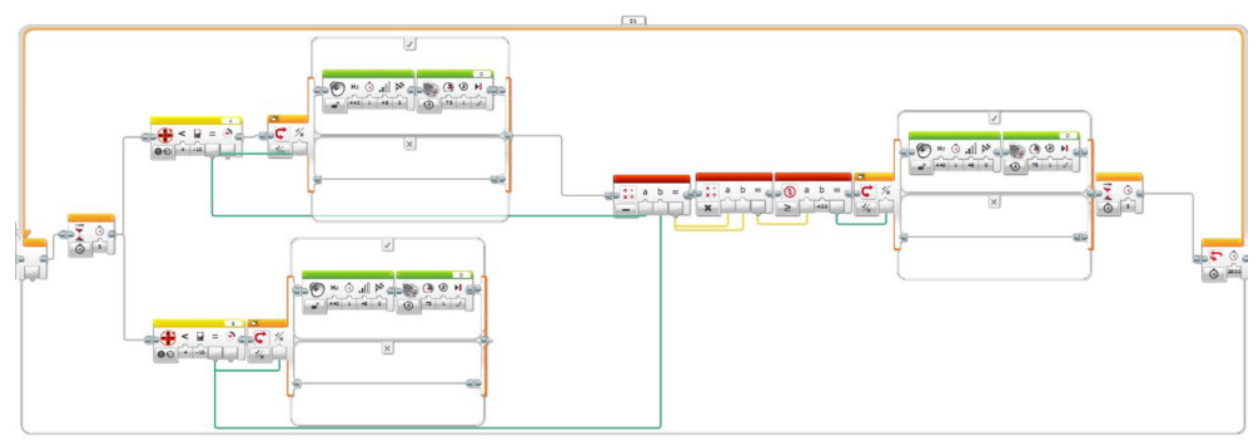

Figure 9. Sitting Posture Monitoring Partial Procedure

\subsection{Active alert device for people with improper sitting posture (desktop)}

The module used in this direction to design the product prototype is an ultrasonic sensor, and the product prototype is shown in Figure 10.

Place the product on a desktop, set the ultrasonic sensor in beacon mode, and use the infrared remote control to start the program. The product will calibrate the standard sitting posture according to the body standards of different people, and use the ultrasonic sensor to measure the distance of the user's head to determine whether it is in the standard sitting posture. When the difference between the user's head distance and the set initial sitting head distance is greater than $(20 \mathrm{~cm})$, the product will detect the distance change and give an alert. If the incorrect sitting posture is not changed in $10 \mathrm{~s}$, the product will continue to continuously remind the user of the incorrect sitting posture. The program display diagram is shown in Figure 11.

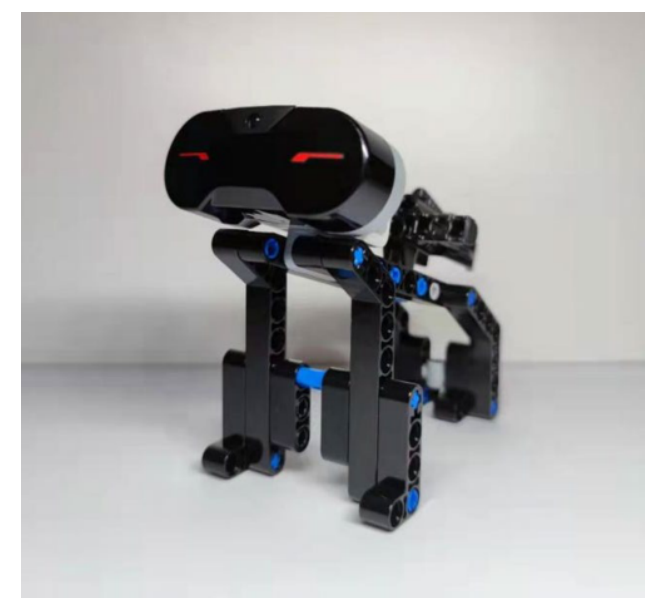

Figure 10. Ultrasonic distance measurement product prototype 


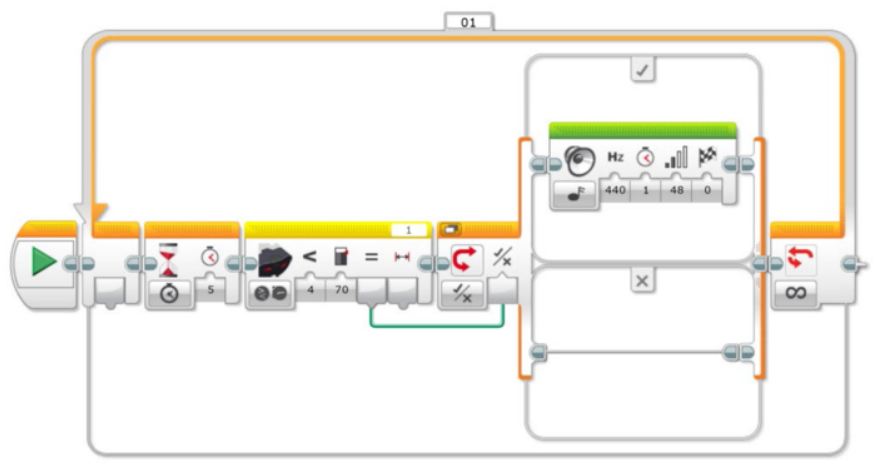

Figure 11. Ultrasonic distance measurement and alerting program demonstration diagram

\subsection{Active alert device for people with improper sitting posture (integral)}

The above product also has an anti-sitting function. When the user has been sitting in the chair for an hour, a beep will sound to tell the user that it is time to take a break. At this time, the product will also enter standby mode, and when the user has rested, the machine can be turned back on to continue a new round of testing. The procedure is shown in Figures 12 and 13.

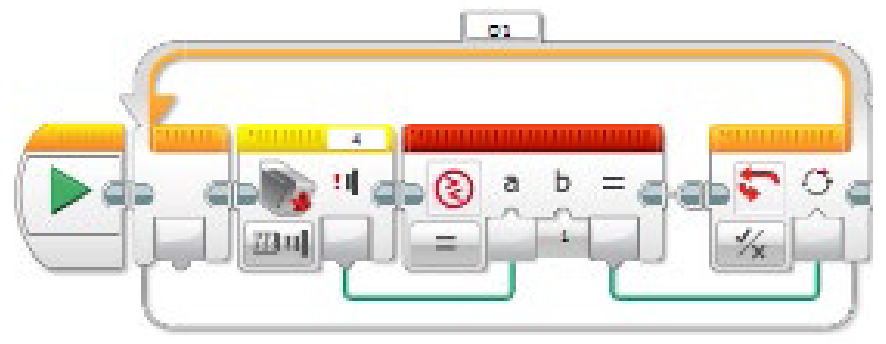

Figure 12. Start side of the anti-sedentary program

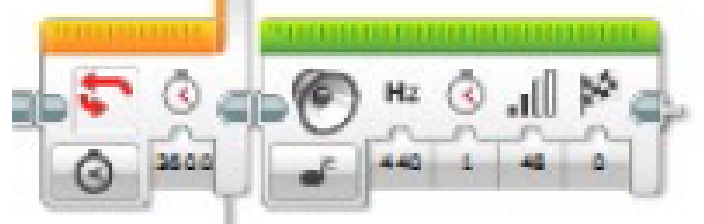

Figure 13. End side of the anti-sedentary program 


\section{Conclusion}

This paper completes the analysis of the problem of improper sitting and the analysis of the existing product situation, and then makes some scenario ideas, analysis and refinement. Through classroom observation, researchers found this Intelligent product can effectively solve a variety of sitting posture problems and improve learners' learning efficiency.

This design aims to use LEGO EV3 component as a platform to design an intelligent sitting correction device based on the existing problem of poor sitting posture through intelligent and scientific means to remind and help people to correct improper sitting posture and maintain physical health. The device has three sets of angle sensors located at the elbow, crotch and knee to detect whether the user's sitting posture is proper or not, and the split structure reduces the burden of the wearable device. The desktop ultrasonic terminal not only provides a more accurate control of sitting posture, but also a timer function to remind users of the dangers of sedentary and excessive eye use.

In future products, we hope to improve the structure to make it more comfortable to wear, and to use angle sensors instead of motors to make the limbs more flexible. In addition, the shape of the device could be made more aesthetically pleasing and ergonomic.

\section{Acknowledgement}

This paper is sponsored by National College Student Innovation and Entrepreneurship Training Program "Design of innovative products Based on EV3" (2021-No.19), and Teaching Reform key project of Beijing Institute of Fashion Technology "Classroom Observation and Analysis of College Basic Courses based on COUPS Scale” (ZDJG-1712).

\section{References}

[1] Feng Shi p. Sedentary injury to the waist, avoid improper sitting posture [J]Teaching reform key project of Beijing Institute of Fashion Technology "Classroom Observation and Analysis of College Basic Courses based on COUPS Scale” (ZDJG-1712). Jiangsu Health Care,2020(12):43.

[2]Wang Chunyang. Analysis of the current situation of the survey of domestic adolescents' body image[J]. Sports teacher and friend,2018,41(01):44-46.

[3] Doreen. The World Sight Report: Protecting your eyes is urgent [J]. The World Vision Report: The urgent need to protect the eyes[J].

[4] Guo Chunrong, Wei J. Research and reflection on the product design of healthy sitting in the Internet era[J]. Tomorrow's Style, 2019, 000(021):P.1-3.

[5] You Qianzi. Research and practice on the design of intelligent cushion system for school-age children [D]. China Academy of Art, 2015.

[6] Hong Pu. Research on ergonomic design and evaluation index system of office chair [D]. Zhengzhou University,2019.

[7] Sun Yueli. Reading with pleasure, starting from the correct reading posture [J]. Contemporary Students,2020(18):20.

[8] Gao Hua-lan. Sitting correctly and exercising more for a healthy study and good life[J]. Adolescent Health,2021,19(10):42-43.

[9] Exploring LEGO EV3 [J]. China Science and Technology Education,2020(01):80. 